\title{
Novel strategies of protecting non-cancer cells during chemotherapy: Are they ready for clinical testing?
}

\section{Zbigniew Darzynkiewicz}

${ }^{1}$ Brander Cancer Research Institute and Department of Pathology, New York Medical College, Valhalla, NY, 10595

Commentary on: Apontes et al. Oncotarget 2011; 2: this issue

Received: March 25, 2011, Accepted: March 28, 2011,

Published: March 29, 2011

Copyright: @ Darzynkiewicz This is an open-access article distributed under the terms of the Creative Commons Attribution License, which permits unrestricted use, distribution, and reproduction in any medium, provided the original author and source are credited.

Chemotherapy and radiotherapy are the most effective anticancer modalities. However the effectiveness of chemotherapy, which targets DNA replication and mitosis, is limited by the side effects resulting from killing normal cells that proliferate. The discovery of molecular abnormities associated with neoplastic growth stimulated attempts to develop targeted therapies aimed at selectively inhibiting growth of cancer by interfering with specific molecules or signal transduction pathways that are over-expressed (overactive) in cancer cells. It was anticipated that targeted cancer therapies would be more effective than current treatments and less harmful to normal cells [1]. Imatinib mesylate (Gleevec, STI-571), the drug developed to treat Philadelphia chromosome positive CML, exemplifies the first successful application of targeted therapy [2].The success, however, is due to the fact that the target (Bcr-Abl tyrosine kinase) fulfils two criteria: (i) is unique to this leukemia and not present in normal cells, and (ii) is essential for cell growth. Most other tumors do not express such unique features and therefore targeted treatments, especially of patients with most common solid tumors, has been less successful and is frequently disappointing [3]. This is not surprising because the target is usually the component of a cell signaling pathway that, while over-expressed in cancer, is essential for the survival of cancer as well as normal cells. For example, if a cancer cell has e.g. $10^{7}$ - and normal cell $10^{3}$ - such cell signaling molecules, the treatment with the targeting inhibitor that inactivates $99.99 \%$ of these molecules will completely deplete the normal cells but leave the cancer cells still with $10^{3}$ molecules. Thus, although the initial cancer response to the inhibitor may be quite dramatic, the escalation of dose and treatment duration is expected to lead to preferential killing of normal rather than cancer cells. Given the above, chemo- and radiotherapy are likely to remain the major therapeutic approaches in the armamentarium of oncologist for the near future.

As mentioned, the side effects of chemotherapy limit its effectiveness. To overcome this limitation strategies designed to protect non-cancer cells during chemotherapy have been proposed. Since chemotherapeutic drugs target DNA replication (DNA damaging agents) or mitosis (mitotic blockers), strategies were aimed to combine chemotherapy with inhibitors that would halt progression of normal cells through the cell cycle, making them drug-resistant during the duration of their arrest [3-6]. In most cancers the regulation of cell cycle progression is impaired, especially as a result of dysfunctional p53 or retinoblastoma pathway. Therefore, the inhibitors targeting these pathways cannot effectively stop the cell cycle of cancer cells and therefore cannot offer protection to cancer cells. While such strategies, defined as cyclotherapy $[5,6]$. have obvious rationale, their implementation in the clinic was difficult, being delayed by the need for extensive clinical trials of the cell cycle inhibitors (e.g. inhibitors of Cdks) as some of them may show toxicity. Furthermore, a highly balanced equilibrium between the protective- versus chemotherapeutic- agents, both in terms of their respective concentrations as well as the sequence of and length of administration, must be tested to achieve successful clinical application.

In the current article in Oncotarget, Apontes et al [7] describe advances that move forward the potential clinical applications of cyclotherapy. The authors used mitotic inhibitors, paclitaxel and nocodazole as the chemotherapeutic agents designed to kill cancer cells. The strategy of normal cells protection relied on the use of either non-genotoxic inducer of $\mathrm{p} 53$, nutlin-3a (N-3a), the inhibitor of mTOR pathway rapamycin (RAPA), or the widely prescribed anti-diabetic drug, possibly affecting IGF-1 signaling, metformin (MF), each tested alone and in combination. Their data are very encouraging. Specifically, the authors have seen that N-3a, RAPA or MF, particularly when applied in combinations, halted cell cycle progression of the three normal human cell lines cells, arresting them reversibly in $\mathrm{G}_{1}$ and/or $\mathrm{G}_{2}$ and thereby protecting from the toxicity of mitotic inhibitors. No such arrest was observed in the case of breast cancer MDA-MB-231 cells having mutant p53. Of importance was the observation that the arrest of normal cells was achieved: (i) for the duration equivalent of the time 
interval during which the treatment of cancer cells with mitotic inhibitors (3 days) eliminated their capability to proliferate (assessed 6 days later); and (ii) the arrest was to a large degree reversible and showed no evident toxicity. The maximal protective effects were seen in drug combinations such as N-3a+RAPA, N-3a+RAPA+MF, or RAPA+MF. Of further interest was the observation that while the protective effect of RAPA + MF for normal cells was seen at the reduced concentration of glucose such conditions were actually cytotoxic for cancer cells. The authors offer specific recommendations on timing and sequence of administration of protective agents versus mitotic inhibitors in treatment of cancer.

Several implications advancing clinical use of cyclotherapy stem from the findings of Apontes et al. ${ }^{7}$ One is that the protective agents MF and RAPA, albeit for different applications, are already widely used in the clinic. Therefore their toxicity and pharmacokinetics are well characterized. N-3a, while not advanced in clinical trials, appears to be mimicked in terms of its ability to activate p53, by low concentrations of actinomycin D $[6,8]$, the drug whose clinical application is also recognized. The agents that can be used to protect normal cells thus have been already clinically tested. Another observation of clinical importance stems from the use of metformin. While metformin was shown to have the property to protect normal cells it also has antineoplastic activity against prostate,[9] breast [10] and other cancers [11]. Application of MF+RAPA, perhaps combined with fasting [12] or with 2-deoxyglucose [12] (2-DG) to reduce glucose utilization (2-DG is also well characterized for clinical use) may provide concurrently the protection of normal cells and the antineoplastic activity.

The advantage of cyclotherapy stems from the fact that response of normal cells to the protective agents targeting their signaling and metabolic pathways, which are well characterized, is predictable. In contrast, targeting cancer is uncertain since many of the pathways, often different in various cancers, are dysfunctional. Furthermore, even personalized targeting, after identification of the defective pathways of a given cancer, may be inadequate because of changes that may occur due to tumor progression, in the time interval between tumor sampling and its full characterization.

\section{REFERENCES}

1. Wikipedia, http://en.wikipedia.org/wiki/Targeted_therapy

2. Muller BA. Imatinib and its successors - how modern chemistry has changed drug development. Curr. Pharm. Des. 2009; 15:120-33.

3. Blagosklonny MV, Darzynkiewicz Z. Why Iressa failed? Toward novel use of kinase inhibitors. Canc Biol Ther 2003; 2:137-40

4. Pardee AB. A restriction point for control of normal animal cell proliferation. Proc Natl Acad Sci USA 1974; 71:128690.

5. Blagosklonny MV, Darzynkiewicz Z. Cyclotherapy: Protection of normal cells and unshielding of cancer cells. Cell Cycle 2002; 1: 375-82.

6. Rao B, van Leeuwen IM, Higgins M, Campbel J, Thompson AM, Lane DP, Lain S. Evaluation of and Actinomycin D/ VX-680 aurora kinase inhibitor combination in p53-based cyclotherapy. Oncotarget 2010; 1:639-50.

7. Apontes $\mathrm{P}$, Leontieva OV, Demidenko $\mathrm{ZN}$, Li F, Blagosklonny MV. Exploring long-term protection of normal human fibroblasts and epithelial cells from chemotherapy in cell culture. Oncotarget 2011; 2: this issue

8. Choong ML, Yang H, Lee MA, Lane DP. Specific activation of the $\mathrm{p} 53$ pathway by low dose actinomycin $\mathrm{D}$ : a new route to p53 cyclotherapy. Cell Cycle 2009; 8:2810-8

9. Clements A, Gao B, Yeap SH, Wong MK, Ali SS, Gurney H. Metformin in prostate cancer: two for the price of one. Ann Oncol 2011; Mar18 (Epub)

10. Zhuang Y, Miskimins K. Metformin induces both caspasedependent and poly(ADP-ribose) polymerase-dependent cell death in breast cancer cells. Mol Cancer Res 2011; Mar 11 (Epub)

11. Kourelis TV, Siegel RD. Metformin and cancer: new applications for an old drug. Med Oncol 2011 Feb 8 (Epub)

12. Halicka DH, Ardelt B, Li X, Melamed MR, Darzynkiewicz Z. 2-deoxyglucose enhances sensitivity of human histiocytic U-935 cells to apoptosis induced by Tumor Necrosis Factor. Cancer Res 1995; 55:444-9. 